International Journal of Pure and Applied Mathematics

Volume $100 \quad$ No. $2 \quad 2015,157-167$

ISSN: 1311-8080 (printed version); ISSN: 1314-3395 (on-line version)

url: http://www.ijpam.eu

doi: http://dx.doi.org/10.12732/ijpam.v100i2.1

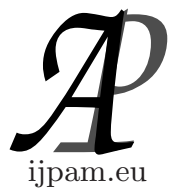

\title{
SOME COMMON FIXED POINT THEOREMS FOR FOUR MAPS IN CONE METRIC SPACES
}

\author{
A.K. Dubey ${ }^{1}{ }^{\S}$, Rita Shukla ${ }^{2}$, Ravi Prakash Dubey ${ }^{3}$ \\ ${ }^{1}$ Department of Mathematics \\ Bhilai Institute of Technology \\ Bhilai House, Durg, 491001, Chhattisgarh, INDIA \\ ${ }^{2}$ Department of Mathematics \\ Shri Shankracharya College of Engineering and Technology \\ Bhilai, 490020, Chhattisgarh, INDIA \\ ${ }^{3}$ Department of Mathematics \\ Dr. C.V. Raman University \\ Bilaspur, Chhattisgarh, INDIA
}

\begin{abstract}
The purpose of this paper is to prove existence of coincidence points and a common fixed point theorems for four maps in cone metric spaces.
\end{abstract}

AMS Subject Classification: 47H10, 54H 25

Key Words: coincidence point, common fixed point, cone metric space

\section{Introduction and Preliminaries}

In 2007, Huang and Zhang (see [1]) introduced the concept of cone metric space, replacing the set of real numbers by an ordered Banach space and proved some fixed point theorems. Subsequently, many authors have studied common fixed point theorems in cone metric spaces (see Abbas et al. (see [3]), Abbas and Rhoades (see [4]) and Rezapour and Hamlbarani (see [2])). Recently, Guang

Received: September 21, 2013

(C) 2015 Academic Publications, Ltd.

$\S$ Correspondence author url: www.acadpubl.eu 
Xing Song et al (see [6]) have obtained coincidence points and common fixed point theorems for two mappings in cone metric spaces. After that Rangamma and Prudhvi (see [9]) generalized and extended the results of (see [6]) for four self-mappings. In this paper we improves and extend the results of (see [6]) and (see [9]).

Before proving our main results first we recall basic definitions and known results that are needed in the sequel.

Definition 1.1. (see [1]) Let $E$ be a real Banach space and $P$ be a subset of $E$. The set $P$ is called a cone if and only if:

(i) $P$ is closed, non-empty, and $P \neq\{0\}$;

(ii) $a, b \in R, a, b \geqslant 0$ and $x, y \in P \Rightarrow a x+b y \in P$;

(iii) $x \in P$ and $-x \in P \Rightarrow P \cap(-P)=\{o\}$.

For a given cone $P \subseteq E$, a partial ordering is defined as $\leqslant$ on $E$ with respect to $P$ by $x \leqslant y$ if and only if $y-x \in P$. It is denoted as $x<y$ to indicate that $x \leqslant y$ but $x \neq y$, while $x \ll y$ will stand for $y-x \in$ int $P$, where int $P$ denotes the interior of $P$.

The cone $P \subset E$ is called normal if there is a number $K>0$ such that, for all $x, y \in E, 0 \leqslant x \leqslant y$ implies $\|x\| \leqslant K\|y\|$.

The least positive number $K$ is called the normal constant of $P$. There are non normal cones also.

In the following, we always suppose $\mathrm{E}$ is a Banach space, $\mathrm{P}$ is a cone in $\mathrm{E}$ with Int $P \neq \phi$ and $\leqslant$ is partial ordering with respect to $P$.

Definition 1.2. (see [1]) Let $X$ be a non-empty set of $E$. Suppose that the map $d: X \times X \rightarrow E$ satisfies:

(i) $0 \leqslant d(x, y)$ for all $x, y \in X$ and $d(x, y)=0$ if and only if $x=y$;

(ii) $d(x, y)=d(y, x)$ for all $x, y \in X$;

(iii) $d(x, y) \leqslant d(x, z)+d(z, y)$ for all $x, y, z \in X$.

Then $d$ is called a cone metric on $X$, and $(X, d)$ is called a cone metric space. It is obvious that the cone metric spaces generalize metric spaces.

Definition 1.3. Let $(X, d)$ be a cone metric space. We say that $\left\{x_{n}\right\}$ is:

(i) a Cauchy sequence if for every $c$ in $E$ with $o \ll c$, there is $N$ such that for all $n, m>N, d\left(x_{n}, x_{m}\right) \ll c$; 
(ii) a convergent sequence if for any $o \ll c$, there is an $N$ such that for all $n>$ $N, d\left(x_{n}, x\right) \ll c$, for some fixed $x$ in $X$. We denote this $x_{n} \rightarrow x(n \rightarrow \infty)$.

A cone metric space $X$ is said to be complete if every Cauchy sequence in $X$ is convergent in $X$.

Definition 1.4. Let $E$ be a real Banach space. For the mappings $f, g$ : $X \rightarrow X$, let $c(f, g)$ denotes set of coincidence points of $f, g$ that is $c(f, g):=$ $\{z \in X: f z=g z\}$.

Definition 1.5. Let $f, g: X \rightarrow X$. Then the pair $(f, g)$ is said to be (IT)-commuting at $z \in X$ if $f(g(z))=g(f(z))$ with $f(z)=g(z)$.

\section{Common Fixed Point Theorems}

In this section we obtain existence of coincidence points and a common fixed point theorems for four self-mappings defined on cone metric space.

Theorem 2.1. Let $(X, d)$ be a cone metric space and $P$ a normal cone with normal constant $K$. Suppose that the mappings $S, T, f$ and $g$ are four self-maps on $X$ such that $S(X)=T(X)$ and $T(X) \subset f(X), S(X) \subset g(X)$ and satisfy the condition

$$
\begin{aligned}
d(S x, T y) \leqslant \alpha d(f x, g y)+\beta[d(f x, T y)+d(g y, S x)] & \\
& +\gamma[d(f x, S x)+d(g y, T y)],
\end{aligned}
$$

for all $x, y \in X$ and $\alpha, \beta, \gamma \in[0,1)$ with $\alpha+2 \beta+2 \gamma<1$.

If $S(X)$ or $T(X)$ is a complete subspace of $X$. Then the maps $(S, f)$ and $(T, g)$ have a coincidence point in $X$. Moreover if $(S, f)$ and $(T, g)$ are (IT)commuting at $p$, then $S, T, f$ and $g$ have a unique common fixed point.

Proof. Suppose $x_{o}$ is an arbitrary point of $X$ and define the sequence $\left\{y_{n}\right\}$ in $X$ such that $y_{2 n}=S x_{2 n}=g x_{2 n+1}$ and $y_{2 n+1}=T x_{2 n+1}=f x_{2 n+2}$.

For all $n=0,1,2, \ldots$ using equation (1), we obtain

$$
\begin{aligned}
d\left(y_{2 n}, y_{2 n+1}\right)= & d\left(S x_{2 n}, T x_{2 n+1}\right) \\
\leqslant & \alpha d\left(f x_{2 n}, g x_{2 n+1}\right)+\beta\left[d\left(f x_{2 n}, T x_{2 n+1}\right)+d\left(g x_{2 n+1}, S x_{2 n}\right)\right] \\
& +\gamma\left[d\left(f x_{2 n}, S x_{2 n}\right)+d\left(g x_{2 n+1}, T x_{2 n+1}\right)\right] \\
\leqslant & \alpha d\left(y_{2 n-1}, y_{2 n}\right)+\beta\left[d\left(y_{2 n-1}, y_{2 n+1}\right)\right]
\end{aligned}
$$




$$
\begin{aligned}
& +\gamma\left[d\left(y_{2 n-1}, y_{2 n}\right)+d\left(y_{2 n}, y_{2 n+1}\right)\right] \\
\leqslant & \alpha d\left(y_{2 n-1}, y_{2 n}\right)+\beta\left[d\left(y_{2 n-1}, y_{2 n}\right)+d\left(y_{2 n}, y_{2 n+1}\right)\right] \\
& +\gamma\left[d\left(y_{2 n-1}, y_{2 n}\right)+d\left(y_{2 n}, y_{2 n+1}\right)\right] \\
= & (\alpha+\beta+\gamma) d\left(y_{2 n-1}, y_{2 n}\right)+(\beta+\gamma) d\left(y_{2 n}, y_{2 n+1}\right) \\
d\left(y_{2 n}, y_{2 n+1}\right) \leqslant & \frac{\alpha+\beta+\gamma}{1-\beta-\gamma} d\left(y_{2 n-1}, y_{2 n}\right) \\
d\left(y_{2 n}, y_{2 n+1}\right) \leqslant & \delta d\left(y_{2 n-1}, y_{2 n}\right)
\end{aligned}
$$

where $\delta=\frac{\alpha+\beta+\gamma}{1-\beta-\gamma}<1$, since $\alpha+2 \beta+2 \gamma<1$.

Similarly, it can be shown that

$$
d\left(y_{2 n+1}, y_{2 n+2}\right) \leqslant \delta d\left(y_{2 n}, y_{2 n+1}\right)
$$

Therefore, for all $n$

$$
d\left(y_{n+1}, y_{n+2}\right) \leqslant \delta d\left(y_{n}, y_{n+1}\right) \leqslant \cdots \leqslant \delta^{n+1} d\left(y_{o}, y_{1}\right)
$$

Now, for any $m>n$

$$
\begin{aligned}
d\left(y_{n}, y_{m}\right) & \leqslant d\left(y_{n}, y_{n+1}\right)+d\left(y_{n+1}, y_{n+2}\right)+\cdots+d\left(y_{m-1}, y_{m}\right) \\
& \leqslant\left[\delta^{n}+\delta^{n+1}+\cdots+\delta^{m-1}\right] d\left(y_{1}, y_{o}\right) \\
& \leqslant \frac{\delta^{n}}{1-\delta} d\left(y_{1}, y_{o}\right)
\end{aligned}
$$

By the definition of normal cone:

$$
\left\|d\left(y_{n}, y_{m}\right)\right\| \leqslant\left(\frac{\delta^{n}}{1-\delta}\right) K\left\|d\left(y_{1}, y_{o}\right)\right\|
$$

which implies that $d\left(y_{n}, y_{m}\right) \rightarrow 0$ as $n, m \rightarrow \infty$.

Hence $\left\{y_{n}\right\}$ is a Cauchy sequence. Since $\left\{y_{n}\right\}$ is a Cauchy sequence in $T(X)$ which is complete there exists $z \in T(X)$ such that $y_{n} \rightarrow z$. Since $T(X) \subset f(X)$, then there exists a point $u \in X$ such that $z=f u$. Let us prove that $z=S u$.

Then by the triangle inequality and (1), we have

$$
\begin{aligned}
d(S u, z) \leqslant & d\left(S u, T x_{2 n-1}\right)+d\left(T x_{2 n-1}, z\right) \\
\leqslant & \alpha d\left(f u, g x_{2 n-1}\right)+\beta\left[d\left(f u, T x_{2 n-1}\right)+d\left(g x_{2 n-1}, S u\right)\right] \\
& +\gamma\left[d(f u, S u)+d\left(g x_{2 n-1}, T x_{2 n-1}\right)\right]+d\left(T x_{2 n-1}, z\right) .
\end{aligned}
$$

By the definition of normal cone:

$$
\|d(S u, z)\| \leqslant K\left(\alpha\left\|d\left(f u, g x_{2 n-1}\right)\right\|+\beta\left[\left\|d\left(f u, T x_{2 n-1}\right)\right\|\right.\right.
$$




$$
\begin{aligned}
& \left.+\left\|d\left(g x_{2 n-1}, S u\right)\right\|\right]+\gamma[\|d(f u, S u)\| \\
& \left.\left.+\left\|d\left(g x_{2 n-1}, T x_{2 n-1}\right)\right\|\right]+\left\|d\left(T x_{2 n-1}, z\right)\right\|\right) .
\end{aligned}
$$

Letting $n \rightarrow \infty$, we obtain

$$
\begin{aligned}
d(S u, z) \leqslant & \alpha d(z, z)+\beta[d(z, z)+d(z, S u)] \\
& +\gamma[d(z, S u)+d(z, z)]+d(z, z) \\
\leqslant & (\beta+\gamma) d(z, S u)
\end{aligned}
$$

which is a contradiction, since $\alpha+2 \beta+2 \gamma<1$. So $S u=z$.

Therefore

$$
z=S u=f u, u \text { is a coincidence point of }\{S, f\} .
$$

Since, $S(X) \subseteq g(X)$ there exists a point $\vartheta \in X$ such that $z=g \vartheta$. We shall show that $T \vartheta=z$. Now from (1), we have

$$
\begin{aligned}
d(z, T \vartheta)= & d(S u, T \vartheta) \\
\leqslant & \alpha d(f u, g \vartheta)+\beta[d(f u, T \vartheta)+d(g \vartheta, S u)] \\
& +\gamma[d(f u, S u)+d(g \vartheta, T \vartheta)] \\
\leqslant & \alpha d(z, z)+\beta[d(z, z)+d(z, T \vartheta)] \\
& +\gamma[d(z, z)+d(z, T \vartheta)] \\
\leqslant & (\beta+\gamma) d(z, T \vartheta),
\end{aligned}
$$

which is a contradiction, since $\alpha+2 \beta+2 \gamma<1$. So $z=T \vartheta$, Therefore

$$
z=T \vartheta=g \vartheta, \vartheta \text { is a coincidence point of }\{T, g\}
$$

From (2) and (3) it follows

$$
S u=f u=T \vartheta=g \vartheta(=z) .
$$

Since, $(S, f),(T, g)$ are (IT)-commuting

$$
\begin{aligned}
d(S S u, S u)= & d(S S u, f u) \\
= & d(S S u, T \vartheta) \\
\leqslant & \alpha d(f S u, g \vartheta)+\beta[d(f S u, T \vartheta)+d(g \vartheta, S S u)] \\
& +\gamma[d(f S u, S S u)+d(g \vartheta, T \vartheta)] \\
\leqslant & \alpha d(S f u, g \vartheta)+\beta[d(S f u, T \vartheta)+d(S S u, g \vartheta)]
\end{aligned}
$$




$$
\begin{aligned}
& +\gamma[d(S f u, S S u)+d(g \vartheta, T \vartheta)] \\
\leqslant & \alpha d(S S u, S u)+\beta[d(S S u, S u)+d(S S u, S u)] \\
& +\gamma[d(S S u, S S u)+d(z, z)] \\
\leqslant & \alpha d(S S u, S u)+\beta[2 d(S S u, S u)] \\
\leqslant & (\alpha+2 \beta) d(S S u, S u)
\end{aligned}
$$

which is a contradiction, since $\alpha+2 \beta+2 \gamma<1$.

Therefore

$$
\begin{gathered}
S S u=S u(=z), \\
S S u=S u=S f u=f S u \quad(\text { since } c(S, f) \text { is }(\text { IT }) \text {-commuting }), \\
S S u=f S u=S u(=z),
\end{gathered}
$$

or

$$
S u=z \text { is a common fixed point of } S \text { and } f
$$

Similarly

$$
\begin{aligned}
& T \vartheta=T T \vartheta=T g \vartheta=g T \vartheta \\
& \Rightarrow T T \vartheta=g T \vartheta=T \vartheta(=z) .
\end{aligned}
$$

Therefore

$$
T v(=z) \text { is a common fixed point of } T \text { and } g .
$$

In view of (5) and (6) it follows that $S, T, f$ and $g$ have a common fixed point namely $z$.

Uniqueness. Let $z_{1}$ be another common fixed point of $S, T, f$ and $g$. Then

$$
\begin{aligned}
d\left(z, z_{1}\right)= & d\left(S z, T z_{1}\right) \\
\leqslant & \alpha d\left(f z, g z_{1}\right)+\beta\left[d\left(f z, T z_{1}\right)+d\left(g z_{1}, S z\right)\right] \\
& +\gamma\left[d(f z, S z)+d\left(g z_{1}, T z_{1}\right)\right] \\
\leqslant & \alpha d\left(z, z_{1}\right)+\beta\left[d\left(z, z_{1}\right)+d\left(z_{1}, z\right)\right] \\
& +\gamma\left[d(z, z)+d\left(z_{1}, z_{1}\right)\right] \\
\leqslant & (\alpha+2 \beta) d\left(z, z_{1}\right)
\end{aligned}
$$

which is a contradiction, since $\alpha+2 \beta+2 \gamma<1$. Hence $z=z_{1}$ and moreover, $S, T, f$ and $g$ have a unique common fixed point. 
Theorem 2.2. Let $(X, d)$ be a cone metric space and $P$ a normal cone with normal constant $K$. Suppose that the mappings $S, T, f$ and $g$ are four self-maps on $X$ such that $S(X)=T(X)$ and $T(X) \subset f(X), S(X) \subset g(X)$ and satisfy the condition

$$
\begin{aligned}
d(S x, T y) \leqslant \alpha d(f x, g y)+\beta[d(f x, S x)+d(f x, T y)] & \\
& +\gamma[d(g y, S x)+d(g y, T y)],
\end{aligned}
$$

for all $x . y \in X$ and $\alpha, \beta, \gamma \in[0,1)$ with $\alpha+2 \beta+2 \gamma<1$.

If $S(X)$ or $T(X)$ is a complete subspace of $X$. Then the maps $(S, f)$ and $(T, g)$ have a coincidence point in $X$. Moreover if $(S, f)$ and $(T, g)$ are (IT)commuting at $p$, then $S, T, f$ and $g$ have a unique common fixed point.

Proof. Suppose $x_{o}$ is an arbitrary point of $X$ and define the sequence $\left\{y_{n}\right\}$ in $X$ such that $y_{2 n}=S x_{2 n}=g x_{2 n+1}$ and $y_{2 n+1}=T x_{2 n+1}=f x_{2 n+2}$, for all $n=0,1,2, \ldots$.

By the equation (8), we have

$$
\begin{aligned}
d\left(y_{2 n}, y_{2 n+1}\right)= & d\left(S x_{2 n}, T x_{2 n+1}\right) \\
\leqslant & \alpha d\left(f x_{2 n}, g x_{2 n+1}\right)+\beta\left[d\left(f x_{2 n}, S x_{2 n}\right)+d\left(f x_{2 n}, T x_{2 n+1}\right)\right] \\
& +\gamma\left[d\left(g x_{2 n+1}, S x_{2 n}\right)+d\left(g x_{2 n+1}, T x_{2 n+1}\right)\right] \\
\leqslant & \alpha d\left(y_{2 n-1}, y_{2 n}\right)+\beta\left[d\left(y_{2 n-1}, y_{2 n}\right)+d\left(y_{2 n-1}, y_{2 n+1}\right)\right] \\
& +\gamma\left[d\left(y_{2 n}, y_{2 n}\right)+d\left(y_{2 n}, y_{2 n+1}\right)\right] \\
\leqslant & \alpha d\left(y_{2 n}, y_{2 n-1}\right)+\beta\left[d\left(y_{2 n}, y_{2 n-1}\right)+d\left(y_{2 n-1}, y_{2 n}\right)+d\left(y_{2 n}, y_{2 n+1}\right)\right] \\
& +\gamma\left[d\left(y_{2 n}, y_{2 n+1}\right)\right] \\
\leqslant & (\alpha+2 \beta) d\left(y_{2 n}, y_{2 n-1}\right)+(\beta+\gamma) d\left(y_{2 n}, y_{2 n+1}\right) .
\end{aligned}
$$

Therefore

$$
\begin{gathered}
d\left(y_{2 n}, y_{2 n+1}\right) \leqslant \frac{\alpha+2 \beta}{1-\beta-\gamma} d\left(y_{2 n-1}, y_{2 n}\right), \\
d\left(y_{2 n}, y_{2 n+1}\right) \leqslant \delta d\left(y_{2 n-1}, y_{2 n}\right),
\end{gathered}
$$

where $\delta=\frac{\alpha+2 \beta}{1-\beta-\gamma}<1$, since $\alpha+2 \beta+2 \gamma<1$.

Similarly, it can be shown that

$$
d\left(y_{2 n+1}, y_{2 n+2}\right) \leqslant \delta d\left(y_{2 n}, y_{2 n+1}\right) .
$$

Therefore, for all $n$ :

$$
d\left(y_{n+1}, y_{n+2}\right) \leqslant \delta d\left(y_{n}, y_{n+1}\right) \leqslant \cdots \leqslant \delta^{n+1} d\left(y_{o}, y_{1}\right) .
$$


For any $m>n$

$$
\begin{aligned}
d\left(y_{n}, y_{m}\right) & \leqslant d\left(y_{n}, y_{n+1}\right)+d\left(y_{n+1}, y_{n+2}\right)+\cdots+d\left(y_{m-1}, y_{m}\right) \\
& \leqslant\left[\delta^{n}+\delta^{n+1}+\cdots+\delta^{m-1}\right] d\left(y_{1}, y_{o}\right) \\
& \leqslant \frac{\delta^{n}}{1-\delta} d\left(y_{1}, y_{o}\right) .
\end{aligned}
$$

By definition of Normal cone, we have

$$
\left\|d\left(y_{n}, y_{m}\right)\right\| \leqslant \frac{\delta^{n}}{1-\delta} K\left\|d\left(y_{1}, y_{o}\right)\right\|
$$

which implies that $d\left(y_{n}, y_{m}\right) \rightarrow 0$ as $n, m \rightarrow \infty$.

Hence $\left\{y_{n}\right\}$ is a Cauchy sequence. Since $\left\{y_{n}\right\}$ is a Cauchy sequence in $T(X)$ which is complete there exists $z \in T(X)$ such that $y_{n} \rightarrow z$. Since $T(X) \subset f(X)$, then there exists a point $u \in X$ such that $z=f u$. Let us prove that $z=S u$.

Then by the triangle inequality and (8), we have

$$
\begin{aligned}
d(S u, z) \leqslant & d\left(S u, T x_{2 n-1}\right)+d\left(T x_{2 n-1}, z\right) \\
\leqslant & \alpha d\left(f u, g x_{2 n-1}\right)+\beta\left[d(f u, S u)+d\left(f u, T x_{2 n-1}\right)\right] \\
& +\gamma\left[d\left(g x_{2 n-1}, S u\right)+d\left(g x_{2 n-1}, T x_{2 n-1}\right)\right]+d\left(T x_{2 n-1}, z\right) .
\end{aligned}
$$

By the definition of normal cone

$$
\begin{aligned}
\|d(S u, z)\| \leqslant & K\left(\alpha\left\|d\left(f u, g x_{2 n-1}\right)\right\|+\beta[\|d(f u, S u)\|\right. \\
& \left.+\left\|d\left(f u, T x_{2 n-1}\right)\right\|\right]+\gamma\left[\left\|d\left(g x_{2 n-1}, S u\right)\right\|\right. \\
& \left.\left.+\left\|d\left(g x_{2 n-1}, T x_{2 n-1}\right)\right\|\right]+\left\|d\left(T x_{2 n-1}, z\right)\right\|\right) .
\end{aligned}
$$

Letting $n \rightarrow \infty$, then

$$
\begin{aligned}
d(S u, z) \leqslant & \alpha d(z, z)+\beta[d(z, S u)+d(z, z)] \\
& +\gamma[d(z, S u)+d(z, z)]+d(z, z) \\
d(S u, z) \leqslant & (\beta+\gamma) d(S u, z),
\end{aligned}
$$

which is a contradiction, since $\alpha+2 \beta+2 \gamma<1$.

Therefore $S u=z$ and

$$
z=S u=f u, u \text { is a coincidence point of }\{S, f\} .
$$

Since, $S(X) \subseteq g(X)$ there exists a point $\vartheta \in X$ such that $z=g \vartheta$. We shall show that $T \vartheta=z$. Then by (8), we have

$$
d(z, T \vartheta)=d(S u, T \vartheta)
$$




$$
\begin{aligned}
\leqslant & \alpha d(f u, g \vartheta)+\beta[d(f u, S u)+d(f u, T \vartheta)] \\
& +\gamma[d(g \vartheta, S u)+d(g \vartheta, T \vartheta)] \\
\leqslant & \alpha d(z, z)+\beta[d(z, z)+d(z, T \vartheta)]+\gamma[d(z, z)+d(z, T \vartheta)] \\
d(z, T \vartheta) \leqslant & (\beta+\gamma) d(z, T \vartheta),
\end{aligned}
$$

which is a contradiction, since $\alpha+2 \beta+2 \gamma<1$. The last implies $z=T \vartheta$.

Therefore

$$
z=T \vartheta=g \vartheta, \vartheta \text { is a coincidence point of }\{T, g\}
$$

From (9) and (10) it follows

$$
S u=f u=T \vartheta=g \vartheta(=z) .
$$

Since, $(S, f),(T, g)$ are (IT)-commuting

$$
\begin{aligned}
d(S S u, S u)= & d(S S u, f u) \\
= & d(S S u, T \vartheta) \\
\leqslant & \alpha d(f S u, g \vartheta)+\beta[d(f S u, S S u)+d(f S u, T \vartheta)] \\
& +\gamma[d(g \vartheta, S S u)+d(g \vartheta, T \vartheta)] \\
\leqslant & \alpha d(S f u, g \vartheta)+\beta[d(S f u, S S u)+d(S f u, T \vartheta)] \\
& +\gamma[d(S S u, g \vartheta)+d(g \vartheta, T \vartheta)] \\
\leqslant & \alpha d(S S u, S u)+\beta[d(S S u, S S u)+d(S S u, S u)] \\
& +\gamma[d(S S u, S u)+d(S u, S u)] \\
& d(S S u, S u) \leqslant(\alpha+\beta+\gamma) d(S S u, S u)
\end{aligned}
$$

which is a contradiction, since $\alpha+2 \beta+2 \gamma<1$.

Therefore $S S u=S u(=z)$,

$$
\begin{gathered}
S S u=S u=S f u=f S u \quad(\text { since } c(S, f) \text { is (IT)-commuting), } \\
S S u=f S u=S u(=z) .
\end{gathered}
$$

Hence

$$
S u=z \text {, is a common fixed point of } S \text { and } f \text {. }
$$

Similarly

$$
T \vartheta=T T \vartheta=T g \vartheta=g T \vartheta
$$




$$
\Rightarrow T T \vartheta=g T \vartheta=T \vartheta(=z)
$$

Therefore

$$
T v(=z) \text { is a common fixed point of } T \text { and } g .
$$

In view of (12) and (13) it follows that $S, T, f$ and $g$ have a common fixed point namely $z$.

Uniqueness. Let $z_{1}$ be another common fixed point of $S, T, f$ and $g$. Then

$$
\begin{aligned}
d\left(z, z_{1}\right)= & d\left(S z, T z_{1}\right) \\
\leqslant & \alpha d\left(f z, g z_{1}\right)+\beta\left[d(f z, S z)+d\left(f z, T z_{1}\right)\right] \\
& +\gamma\left[d\left(g z_{1}, S z\right)+d\left(g z_{1}, T z_{1}\right)\right] \\
\leqslant & \alpha d\left(z, z_{1}\right)+\beta\left[d(z, z)+d\left(z, z_{1}\right)\right] \\
& +\gamma\left[d\left(z_{1}, z\right)+d\left(z_{1}, z_{1}\right)\right] \\
\leqslant & \alpha d\left(z, z_{1}\right)+\beta d\left(z, z_{1}\right)+\gamma d\left(z, z_{1}\right) \\
\leqslant & (\alpha+\beta+\gamma) d\left(z, z_{1}\right)
\end{aligned}
$$

which is a contradiction, since $\alpha+2 \beta+2 \gamma<1$. Hence $z=z_{1}$ and moreover, $S, T, f$ and $g$ have a unique common fixed point.

\section{References}

[1] L.G. Huang, X. Zhang, Cone metric spaces and fixed point theorems of contractive mappings, J. Math. Anal.App l., 332, No. 2 (2007), 1468-1476.

[2] S. Rezapour, R. Hamlbarani, Some notes on the paper "Cone metric spaces and fixed point theorem of contractive mappings, J. Math. Anal. Appl., 345 (2008), 719-724.

[3] M. Abbas, G. Jungck, Common fixed point results for non commuting mappings without continuity in cone metric spaces, J. Math. Anal. Appl., 341 (2008), 416-420.

[4] M. Abbas, B.E. Rhoades, Fixed and periodic point results in cone metric spaces, Appl. Math. Lett., 22 (2009), 511-515.

[5] I. Beg, M. Abbas, Coincidence point and invariant approximation for mappings satisfying generalized weak contractive condition, Fixed Point Theory Appl., (2006), 1-7, Article ID 74503. 
[6] Guang Xing Song, X. Huang, Xiaoyan Sun, Yian Zhao, Guotao Wang, New common fixed point theorems for maps on cone metric spaces, Applied Mathematics Letters, 23 (2010), 1033-1037.

[7] B. Fisher, Four mappings with a common fixed point, J. Univ. Kuwait Sci. (1981), 131-139.

[8] V. Berinde, A common fixed point theorem for compatible quasi contractive self mappings in metric spaces, Appl. Math. Comput., 213 (2009), 348-354.

[9] M. Rangamma, K. Prudhvi, A Common fixed point theorem for four maps in cone metric spaces, Int. J. of Pure and Applied Mathematics, 78, No. 5 (2012), 797-803. 
